\title{
Strength profile in young male athletes from different sports
}

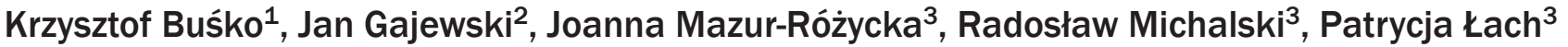 \\ ${ }^{1}$ Institute of Physical Culture, Kazimierz Wielki University, Bydgoszcz, Poland; ${ }^{2}$ Department of Statistics and Informatics, \\ Józef Piłsudski University of Physical Education, Warsaw, Poland; ${ }^{3}$ Department of Biomechanics, Institute of Sport, \\ Warsaw, Poland
}

\section{Summary}

Study aim: The aim of the present study was to determine a strength profile which characterizes young male athletes from different sports and to use the method of allometry to synthetically evaluate the muscular strength with respect to body mass.

Material and methods: The study included 85 men who practiced taekwondo ( 8 subjects), 20 judoists, 10 weightlifters, 35 canoeists and 12 speed skaters. Measurements of maximal muscle torques in 10 groups of flexors and extensors of the elbow, shoulder, hip, knee and trunk were carried out in a testing station for muscle torque measurements under static conditions. In order to determine the relationships between the body mass and muscle torques in each muscle group, the authors used a procedure of linear regression for the data expressed as logarithms. Hence, the allometric relationship scaled with body mass was obtained.

Results: The study demonstrated that the mean values of logarithms of maximal muscle torques are significantly different for the representatives of individual sports and they depend on the logarithm of body mass. The analysis of residuals was employed for the evaluation of maximal muscle torques. The highest strength profiles were observed in athletes who practice judo and the lowest in those who represented weightlifting and taekwondo.

Conclusion: The authors propose to use an allometric relationship which takes body mass into consideration during evaluation of strength in individual muscle groups, because the values of muscle torques are not in direct proportion to body mass and their mutual proportions change with an increase in body mass.

\section{Keywords: Allometry - Combat athletes - Canoeing - Speed skating - Strength}

\section{Introduction}

Measurements of maximal muscle torques in group muscles under static conditions is a routine method used for determination of muscle force in laboratory testing [4-9, 21]. The analysis predominantly involves the values of force generated by the muscles of upper of lower limb, one body side (upper limb, lower limb and trunk) or both sides. Assessment of muscle force is frequently made by means of the absolute sum and/ or relative sum of muscle torques in the main muscle groups [8]. Mutual relations of maximal muscle forces in individual human muscle groups are defined as a strength profile [3], topography of muscle strength [19] or topography of muscle torques $[6,8]$. Other indexes of the structure of human muscle force include relations between flexors and extensors in individual joints (flexors-extensors index) [9, 10] or left and right side (dominant and non-dominant limb), termed asymmetry index [10, 20]. The factors which 'disturb' proper interpretation might include different contribution of maximal muscle torques in individual muscle groups to their algebraic sum which results from a specific character of a sport and/or calculation of relative values (calculation per body mass kilogram) in the sports with weight categories. According to some authors, the method which levels the inconvenience which results from the effect of body mass on different relationships, e.g. muscular force or sport results, is offered by allometric scaling $[2,12,17,18]$.

The aim of the present study was to determine the strength profile which typifies young male athletes from different sports and to use the method of allometry for a synthetic evaluation of strength with respect to body mass in athletes from different sports. 


\section{Material and methods}

The study, after approval by the Ethics Committee of Institute of Sport in Warsaw, Poland, included 85 men who practice taekwondo, judo, weightlifting, canoeing and speed skating. The characteristics of the subjects included in the study are presented in Table 1.

Measurements of maximal muscle torques in ten muscle groups (flexors and extensors of the elbow, shoulder, hip, knee and trunk) were taken in testing stations for measurements of muscle torques under static conditions [7]. The muscle torques in elbow flexors and extensors were measured in a sitting position. The subject's arm was supported on an armrest. The angle at the arm joint amounted to $90^{\circ}$. The forearm was positioned at a right angle with respect to the arm. The measurements of muscle torque of arm flexors and extensors were taken in a sitting position. The angle of the arm joint during extension was $70^{\circ}$, with this value being $50^{\circ}$ during flexion. The body trunk was in contact with the testing station and was stabilized by the chest pressed against the testing station by an assistant. The muscle torques in knee flexors and extensors and trunk flexors and extensors were measured in sitting position. The angle in hip and knee joints was $90^{\circ}$. The subjects were stabilized at the level of anterior iliac spine and in further part of thigh. Upper limbs were crossed on the subject's chest. Muscles which extended the limb in hip joint were measured in face-up position. The angle at hip joint was $90^{\circ}$. The subject stabilized the trunk, holding the testing station with hands. Maximal extension of a limb in elbow, knee and hip joint was adopted as $0^{\circ}$. In the arm joint, position of the limb along the trunk was adopted as $0^{\circ}$. Position of the trunk in face-up position was adopted as $0^{\circ}$. Rotation axis in the measured joint covered with the rotation axis of the torque meter. Both upper and lower limbs were measured, separately for left and right side, maintaining the order of flexion-extension. The subjects were instructed to develop maximal value of muscle torque.
The analysis used maximal values of muscle torques obtained for trunk and means values of muscle torques for left and right limb.

Because the data did not exhibit a normal distribution, they were subjected to a logarithmic transformation. The multivariate analysis of covariance (MANCOVA) was employed in order to analyse mean values between the groups. A logarithm of body mass was adopted as a covariate. In order to determine the relationship between the body mass and muscle torque in each muscle group, the authors used the analysis of linear regression. The linear regression analysis was based on an allometric relationship scaled with body mass, with regression equation presented in a logarithmic form, and the allometric relationship expressed by the formula (1):

$$
\ln \mathrm{M}_{\mathrm{o}}(\mathrm{m})=\mathrm{a} \ln \mathrm{m}+\mathrm{b} .
$$

After removal of logarithms, the equation 1 is given by the power equation:

$$
\mathrm{M}_{\mathrm{o}}(\mathrm{m})=\mathrm{Bm}^{\mathrm{a}},
$$

where: $\mathrm{M}_{\mathrm{o}}$ - expected torque, $\mathrm{m}$ - body mass, $\mathrm{a}$ and $\mathrm{b}$ regression coefficients, $\mathrm{B}=\mathrm{e}^{\mathrm{b}}$.

The analysis of residuals (the difference between the actual and expected torques) was used in order to evaluate the maximal muscle torques:

$$
\mathrm{d}=\ln \mathrm{M}-\ln \mathrm{M}_{\mathrm{o}} \text {. }
$$

where: $\mathrm{d}$ - residual, $\mathrm{M}$ - actual torque.

In order to evaluate the results of obtained by a subject compared to the group, the authors used normalized residuals, i.e. the residuals divided by standard regression error (4):

$$
\mathrm{d}^{*}=\frac{\ln \mathrm{M}-\ln \mathrm{M}_{\mathrm{o}}}{\mathrm{SE}}=\frac{\mathrm{d}}{\mathrm{SE}},
$$

where: $\mathrm{d}$ - residual, SE - standard regression error,

$\mathrm{SE}=\sqrt{\frac{\sum_{i=1}^{\mathrm{n}} d_{i}^{2}}{\mathrm{n}-2}}, \mathrm{n}-$ number of subjects.

All the calculations were carried out using Statistica software (v. 9.0, StatSoft).

Table 1. Somatic characteristics of the studied groups (Mean values \pm SD)

\begin{tabular}{lccccc}
\hline Sport & Number of Subjects & Age [years] & Body Mass [kg] & Body Height [cm] & Experience [years] \\
\hline Taekwondo & 8 & $15.8 \pm 0.9$ & $62.6 \pm 13.1$ & $178.4 \pm 9.7$ & $7.94 \pm 1.27$ \\
Judo & 20 & $17.5 \pm 1.5$ & $81.5 \pm 20.2$ & $177.2 \pm 7.7$ & $9.45 \pm 2.56$ \\
Weightlifting & 10 & $16.2 \pm 1.2$ & $81.8 \pm 17.9$ & $176.1 \pm 4.6$ & $2.95 \pm 1.12$ \\
Canoeing & 35 & $17.8 \pm 0.9$ & $82.1 \pm 6.2$ & $183.5 \pm 6.1$ & $5.61 \pm 1.56$ \\
Speed Skating & 12 & $18.3 \pm 0.7$ & $76.1 \pm 6.9$ & $179.2 \pm 3.7$ & $5.88 \pm 2.80$ \\
\hline
\end{tabular}




\section{Results}

Mean values $( \pm \mathrm{SD})$ of maximal muscle torques generated by the athletes who practice taekwondo, judo, weightlifting, canoeing and speed skating are presented in Table 2.

Shapiro-Wilk test demonstrated that, after logarithmic transformation of maximal muscle torques and body mass, the distributions of all the variables did not differ significantly from normal distribution $(p>0.05)$. The logarithms of maximal muscle torques correlated significantly $(p<0.001)$ with body mass logarithm (Table 3).

Mean logarithms of maximal muscle torques in individual sports were compared by means of multivariate analysis of covariance. The analysis concerned the relationship of logarithms of maximal muscle forces with the sport (constant factor) with the covariate in the form of body mass logarithm. The study demonstrated a significant effect of the type of sport $\left(\mathrm{F}_{10.65}=12.95\right.$; $p<0.001)$ and body mass logarithm $\left(\mathrm{F}_{50.318}=4.64\right.$; $p<0.001$ ) on the obtained values of maximal muscle torques. The expected values of logarithms of maximal muscle torques, and residuals and normalized residuals (according to the formulae 3 and 4) were calculated for each subject. A strength profile which characterized each subject compared to the population was a vector of the normalized residuals which resulted from regression of logarithms of maximal muscle torques to the logarithm of body mass.

Figure 1 presents mean profiles obtained for men who practice the sports included in the study. The highest strength profile was observed in the athletes who practiced judo. Similar strength profiles (substantial advantage of strength in lower limbs and trunk) were observed in speed skaters and the athletes who practiced taekwondo. Canoeists obtained

Table 2. Mean values $( \pm \mathrm{SD})$ of maximal muscle torques $(\mathrm{MT})$ in flexors $(\mathrm{F})$ and extensors $(\mathrm{E})$ in right limbs, in elbow, arm, hip and knee joints and in trunk flexors and extensors in the athletes who practice taekwondo, weightlifting, canoeing and speed skating

\begin{tabular}{lcccccc}
\hline Joint & & $\begin{array}{c}\text { MT }[\mathrm{N} \cdot \mathrm{m}] \\
\text { Taekwondo } \\
\mathrm{n}=8\end{array}$ & $\begin{array}{c}\text { MT }[\mathrm{N} \cdot \mathrm{m}] \\
\text { Judo } \\
\mathrm{n}=20\end{array}$ & $\begin{array}{c}\text { MT }[\mathrm{N} \cdot \mathrm{m}] \\
\text { Weightlifting } \\
\mathrm{n}=10\end{array}$ & $\begin{array}{c}\text { MT }[\mathrm{N} \cdot \mathrm{m}] \\
\text { Canoeing } \\
\mathrm{n}=35\end{array}$ & $\begin{array}{c}\text { MT [N·m] } \\
\text { Speed Skating } \\
\mathrm{n}=12\end{array}$ \\
\hline Elbow & $\mathrm{F}$ & $57.5 \pm 14.5$ & $85.4 \pm 17.8^{\mathrm{a}}$ & $78.8 \pm 9.6^{\mathrm{a}}$ & $84.7 \pm 12.7^{\mathrm{a}}$ & $72.5 \pm 12.2^{\mathrm{abd}}$ \\
Joint & $\mathrm{E}$ & $36.6 \pm 8.6$ & $64.4 \pm 11.9^{\mathrm{a}}$ & $53.3 \pm 8.6^{\mathrm{ab}}$ & $58.4 \pm 8.6^{\mathrm{abc}}$ & $46.7 \pm 8.6^{\mathrm{abcd}}$ \\
Arm & $\mathrm{F}$ & $50.5 \pm 16.8$ & $79.2 \pm 21.0^{\mathrm{a}}$ & $66.0 \pm 11.1^{\mathrm{ab}}$ & $79.4 \pm 13.9^{\mathrm{ac}}$ & $70.8 \pm 9.9^{\mathrm{abd}}$ \\
Joint & $\mathrm{E}$ & $57.7 \pm 18.3$ & $94.2 \pm 21.0^{\mathrm{a}}$ & $74.4 \pm 12.7^{\mathrm{ab}}$ & $98.2 \pm 15.0^{\mathrm{ac}}$ & $74.4 \pm 10.9^{\mathrm{abd}}$ \\
Knee & $\mathrm{F}$ & $115.1 \pm 26.2$ & $136.7 \pm 26.5^{\mathrm{a}}$ & $141.2 \pm 26.3^{\mathrm{a}}$ & $133.3 \pm 27.2^{\mathrm{a}}$ & $137.8 \pm 20.0^{\mathrm{a}}$ \\
Joint & $\mathrm{E}$ & $252.1 \pm 73.8$ & $329.1 \pm 79.7^{\mathrm{a}}$ & $278.3 \pm 63.8^{\mathrm{b}}$ & $305.3 \pm 53.8^{\mathrm{a}}$ & $323.2 \pm 57.4^{\mathrm{ac}}$ \\
Hip & $\mathrm{F}$ & $98.5 \pm 28.2$ & $157.9 \pm 162.3^{\mathrm{a}}$ & $107.6 \pm 15.7^{\mathrm{b}}$ & $116.4 \pm 17.5^{\mathrm{b}}$ & $117.0 \pm 17.8$ \\
Joint & $\mathrm{E}$ & $450.6 \pm 142.1$ & $586.1 \pm 141.7^{\mathrm{a}}$ & $560.1 \pm 164.8^{\mathrm{a}}$ & $523.0 \pm 97.5^{\mathrm{ab}}$ & $549.5 \pm 92.5^{\mathrm{a}}$ \\
Trunk & $\mathrm{F}$ & $136.3 \pm 35.8$ & $206.9 \pm 39.8^{\mathrm{a}}$ & $168.8 \pm 30.4^{\mathrm{ab}}$ & $210.3 \pm 32.7^{\mathrm{ac}}$ & $217.3 \pm 25.4^{\mathrm{ac}}$ \\
& $\mathrm{E}$ & $401.9 \pm 136.0$ & $608.5 \pm 154.9^{\mathrm{a}}$ & $512.5 \pm 130.7^{\mathrm{ab}}$ & $583.2 \pm 102.1^{\mathrm{ac}}$ & $548.3 \pm 106.0^{\mathrm{a}}$ \\
\hline
\end{tabular}

a - mean values which differ significantly compared to taekwondo, $p<0.05 ;{ }^{\mathrm{b}}$ - mean values which differ significantly compared to judo, $p<0.05 ;^{\mathrm{c}}-$ mean values which differ significantly compared to weightlifting, $p<0.05 ;{ }^{\mathrm{d}}-$ mean values which differ significantly compared to canoeing, $p<0.05$.

Table 3. Correlation coefficients (r) between body mass logarithms and the logarithms of muscle torques for individual muscle groups $(\mathrm{n}=85)$

\begin{tabular}{|c|c|c|c|c|c|c|c|c|c|c|}
\hline & \multicolumn{10}{|c|}{ Muscle Group } \\
\hline & $\mathrm{EF}$ & $\mathrm{EE}$ & $\mathrm{AF}$ & $\mathrm{AE}$ & KF & $\mathrm{KE}$ & $\mathrm{HF}$ & $\mathrm{HE}$ & $\mathrm{TF}$ & $\mathrm{TE}$ \\
\hline $\mathrm{r}$ & 0.785 & 0.659 & 0.689 & 0.711 & 0.633 & 0.609 & 0.600 & 0.618 & 0.617 & 0.642 \\
\hline
\end{tabular}

$\mathrm{EF}$ - elbow joint, flexion, $\mathrm{EE}$ - elbow joint, extension, $\mathrm{AF}$ - arm joint, flexion, $\mathrm{AE}$ - arm joint, extension, $\mathrm{KF}$ - knee joint, flexion, $\mathrm{KE}$ - knee joint, extension, HF - hip joint, flexion, HE - hip joint, extension, TF - trunk, flexion, TE - trunk, extension 


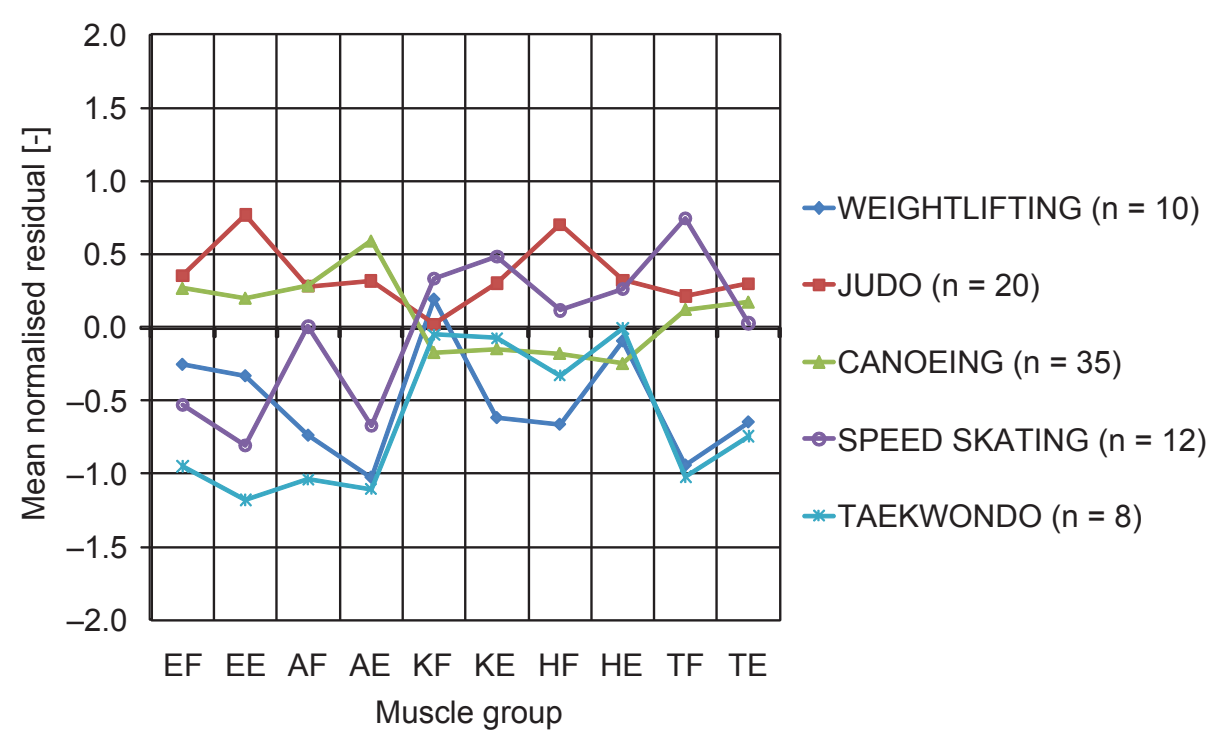

Fig. 1. Mean strength profiles for the sports included in the study (EF - elbow joint, flexion, EE - elbow joint, extension, $\mathrm{AF}$ - arm joint, flexion, $\mathrm{AE}$ - arm joint, extension, $\mathrm{KF}$ - knee joint, flexion, $\mathrm{KE}$ - knee joint, extension, $\mathrm{HF}$ - hip joint, flexion, HE - hip joint, extension, TF - trunk, flexion, TE - trunk, extension)

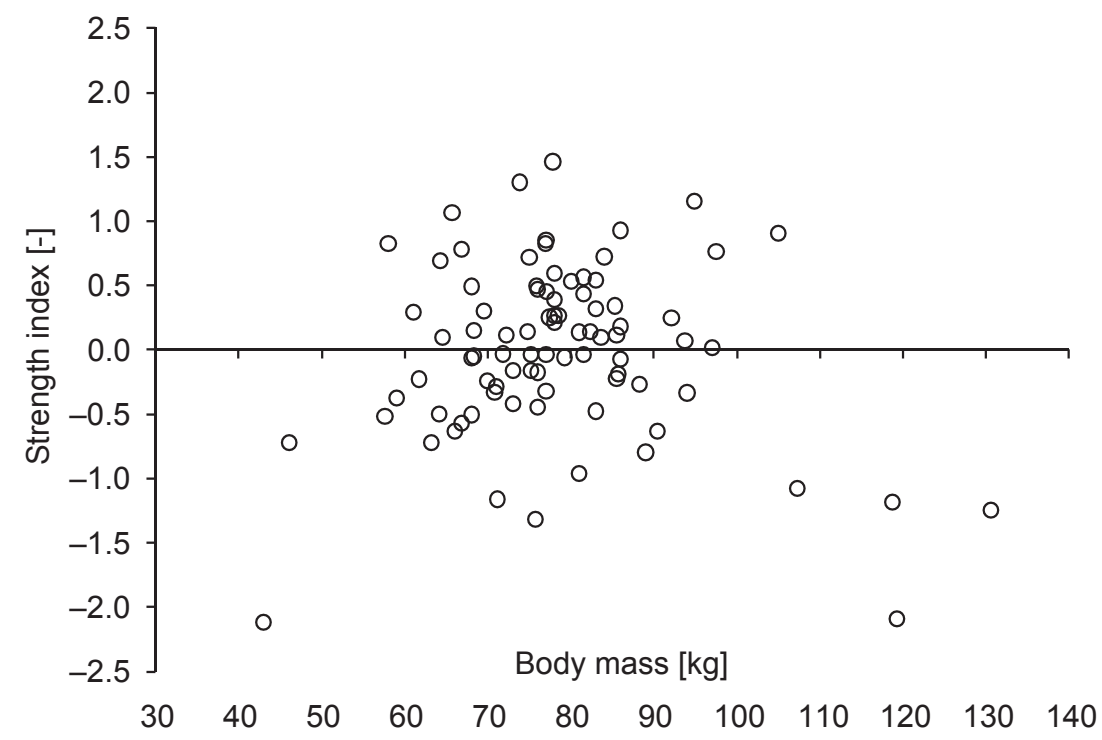

Fig. 2. The strength index (the mean from normalized residuals) vs. body mass for all the subjects tested ( $\mathrm{n}=85)$

the highest values for upper limbs and trunk. The subjects who train weightlifting had relatively low results compared to the athletes who practiced other sports.

In order to synthetically evaluate strength as a motor characteristic, the authors decided to define a strength index as a mean of strength profile components. Fig. 2 presents the correlogram for the index and body mass for all the subjects, whereas Fig. 3 presents the strength indexes for individual groups of men included in the study.

It can be observed that the heaviest subjects (over $115 \mathrm{~kg}$ ) obtained the lowest values of this index.

\section{Discussion}

Recent years have seen an increasing interest in the importance of body size (e.g. body mass and height) as a potential disturbing factor and/or the factor which affects the results of physiological or biomechanical tests. Allometric models are employed in order to eliminate the effect of body size on physiological or biomechanical variables. There are a number of examples presented in the literature of the variables scaled to the differences of body size using allometry, which concerned grip force $[15,22]$, strength and torques in 


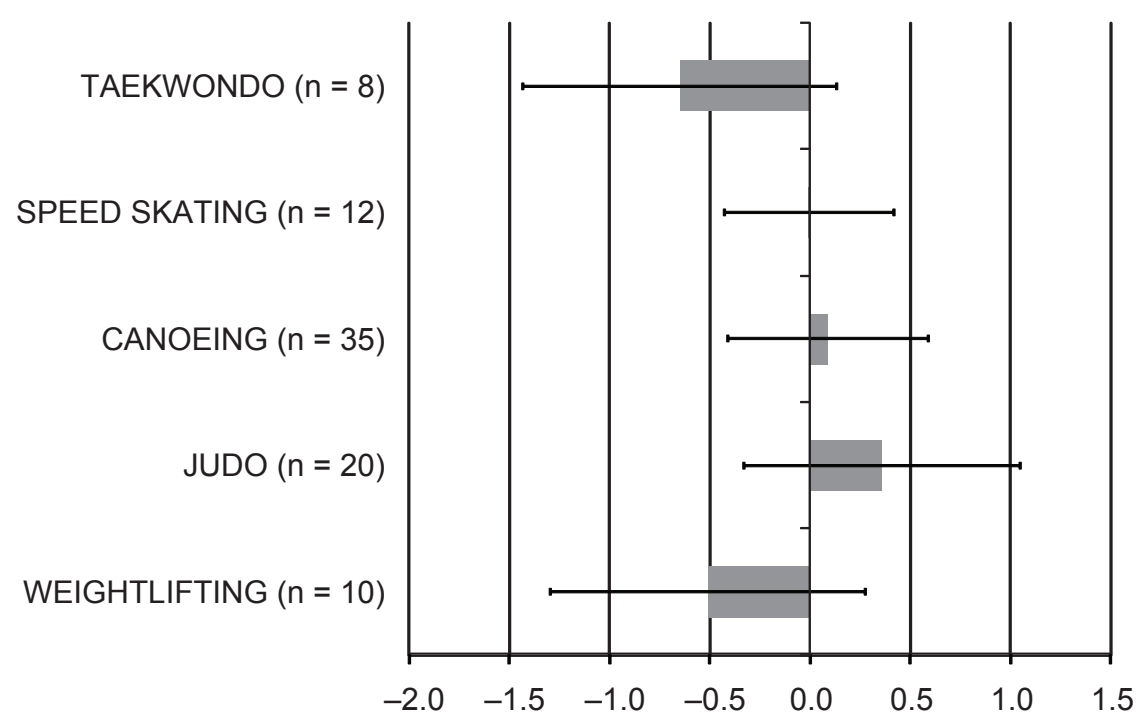

Fig. 3. Mean values of strength index $( \pm S D)$ (calculated for individual subjects as mean values of strength profile) for the representatives of different sports $(n=85)$

flexors and extensors in different joint $[2,12,16]$, maximal force measured by the result of 1 repetition maximal (1RM) [1, 17, 18], height of countermovement jump (CMJ) [16], submaximal and maximal oxygen uptake $[14,19,23]$, rowing speed measured with rowing ergometer at the distance of $2000 \mathrm{~m}$ [14] or peak power and mean power in 30-second supramaximal test performed using upper limbs [13]. A relationship between body size of weightlifters and the obtained results has been discussed in a number of studies. It was assumed that the relationship would be linear or curvilinear. In 1985, Sinclair [17] proposed the use of the method of allometric scaling for comparison of the results obtained by weightlifters from different weight categories. The relationship was described by the second-degree polynomial. Stone et al. [18] used the scaling method for searching for the relationships between maximal force measured with $1 \mathrm{RM}$ squat performance and the results from snatch test in female and male weightlifters. The observations were obtained using Pearson's correlation coefficients, allometric method and Sinclair's method [19]. A significant relationship was found between the result from 1RM squat performance and the results of snatch tests, at the level of 0.84 , independently of body mass and height. Furthermore, the men were stronger than women, even if the body mass and height was eliminated using the scaling methods. Gajewski et al [12] used the allometric model to determine profile of strength the athletes practicing in weightlifting, taekwondo, canoeing and skating fast. Similar profiles were observed in the athletes who practising in speed skating and taekwondo (higher values for lower limbs and trunk), whereas the female subjects who practise canoeing obtained higher values for upper limbs and trunk. In our study, the strength profiles obtained in male athletes practicing the same discipline as Gajewski et al. [12] were similar. Batterham and George [1] utilized the allometric model for searching for the relationships between body mass (weight categories) and the results from weightlifting competitions in consideration of genders. They demonstrated that the allometric model 'punishes' lighter and heavier competitors. Similar results were obtained by Sinclair [17]. According to Batterham and George [1], linear model is not always proper for the analysis of biological relationships. Sometimes, it is more appropriate to use a polynomial or a logarithmic scale. It is generally accepted that a negative impact of high body mass depends on lean body mass. Forbes [11] estimated that the upper limit for lean body mass is $100 \mathrm{~kg}$ for men and $60 \mathrm{~kg}$ for women. These values correspond to body mass of ca. $110-120 \mathrm{~kg}$ in men and $70-80 \mathrm{~kg}$ in women. Increase in body mass over these values might be caused by an increase in the content of fat tissue. In our study, body mass varied from 43.0 to $130.5 \mathrm{~kg}$. The residuals for three extremely heavy subjects (over $115 \mathrm{~kg}$ ) they actually adopted negative values (Fig. 2), which seems to confirm the concerns expressed by Butterham and George [1]. Three heaviest athletes competed in open categories: two in judo and one in weightlifting. They obviously differed from other athletes in their sports in the fat mass. Therefore, it should be found that, in consideration of Forbes's reservations [11], their body build does not match the build which is described by the analysed allometric model. 
In summary, however, one should bear in mind that even if it is possible to find a straight line, determine the correlation coefficient, standard deviation and confidence interval, it still remains merely one of possible mathematical models which quantitatively describe the relationships between the variables. The statistical significance, even if very convincing, does not guarantee a perfect conformity of model and actual results.

\section{Conclusions}

1. Similar strength profiles were found in speed skaters and taekwondo contestants, which might result from the specific character of both sports.

2. The authors propose to use an allometric relationship which takes body mass into consideration during evaluation of strength in individual muscle groups, because the values of muscle torques are not in direct proportion to body mass and their mutual proportions change with an increase in body mass.

3. The mean value of residuals normalized for logarithms of maximal torques in regression to logarithms of body mass for individual muscle groups is recommended to be used as a synthetic strength index.

\section{References}

1. Batterham A., K. George (1997) Allometric modeling does not determine a dimensionless power function ratio for maximal muscular function. J. Apply. Physiol., 83: 2158-2166.

2. Bazzet-Jones D., S. Cobb, N. Joshi Mukta, S. Cashin, J. Earl (2011) Normalizing Hip Muscle Strength: Establishing Body-Size-Independent Measurements. Arch. Phys. Med. Rehab., 92(1): 76-82.

3. Bober T., B. Pietraszewski (1996) Strength of muscle groups in swimmers. Biol. Sport, 13: 155-164.

4. Boguszewska K., D. Boguszewski, K. Buśko (2010) Specjal Judo Fitness Test and biomechanics measurements as a way to control of physical fitness in young judoists. Archives of Budo, 6(4): OA205-209.

5. Buśko K., J. Lewandowska, M. Lipińska, R. Michalski, A. Pastuszak (2013) Somatotype variables related to muscle torque and power output in female volleyball players. Acta Bioeng. Biomech., 15(2): 119-126.

6. Buśko K. (2012) Training-induced changes in the topography of muscle torques and maximal muscle torques in basketball players. Biol. Sport, 29(1): 77-83.

7. Buśko K., J. Gajewski (2011) Muscle strength and power of elite female and male swimmers. BJHPA, 3(1): $13-18$.
8. Buśko K., A. Nowak (2008) Changes of maximal muscle torque and maximal power output of lower extremities in male judoists during training. Hum. Movement, 9(2): 111-115.

9. Buśko K., A. Madej, A. Mastalerz (2008) Changes of muscle torque after sprint and endurance training performed on the cycle ergometer. Biol. Sport, 25(3): 275-294.

10. Drid P., M. Drapsin, T. Trivic, D. Lukač, S. Obadov, Z. Milosevic (2009) Asymmetry of muscle strength in elite athletes. Biomed. Human Kinet., 1: 3-5.

11. Forbes G. B. (1987) Human Body Composition: Growth, Aging, Nutrition and Activity. Springer-Verl., New York.

12. Gajewski J., K. Buśko, J. Mazur, R. Michalski (2011) Application of allometry for determination of strength profile in young female athletes from different sports. Biol. Sport, 28(4): 239-243.

13. Kabitsis C., A.M. Nevill (1992) Power output during arm cycling and its relationship to body size and throwing performance. J. Sports Sci., 10: 568-569.

14. Nevill A.M., S.V. Allen, S.A. Ingham (2011) Modelling the determinants of $2000 \mathrm{~m}$ rowing ergometer performance: a proportional, curvilinear allometric approach. Scand. J. Med. Sci. Sports, 21(1): 73-8. DOI: 10.1111/j.1600-0838.2009.01025.x.

15. Pua Y-H. (2006) Allometric Analysis of Physical Performance Measures in Older Adults. Physical therapy, 86(9): 1263-1270.

16. Pua Y-H., M.T-H. Koh, Y-Y. Teo (2006) Effects of allometric scaling and isokinetics testing methods on the relationship between countermovement jump and quadriceps torque and power. J. Sport Sci., 24(4): 423-432. DOI: 10.1080/02640410500189124

17. Sinclair R.G. (1985) Normalizing the performances of athletes in Olympic Style weightlifting. Can. J. Appl. Sport Sci., 10:94-98.

18. Stone M.H., W.A. Sands, K.C. Pierce, J. Carlock, M. Cardinale, R.U. Newton (2005) Relationship of maximum strength to weightlifting performance. Med. Sci. Sports Exerc., 37(6): 1037-1043.

19. Tartaruga M.P., M.H. de Medeiros, C.L. Alberton, E.L. Cadore, L.A. Peyré-Tartaruga, R.R. Baptista, M. Coertjens, L.F.M. Kruel (2010) Application of the allometric scale for the submaximal oxygen uptake in runners and rowers. Biol. Sport, 27: 297-300.

20. Trzaskoma Z, Ł. Trzaskoma (2001) The proportion between maximal torque of core muscles in male and female athletes. Acta Bioeng. Biomech., 3(Suppl. 2): 601-606.

21. Trzaskoma Z, Ł. Trzaskoma (2006) Structure of maximal muscle strength of lower extremities in highly experienced athletes. Phys. Edu. Sport, 50: 73-78. 
22. Vanderburgh P.M., M.T. Mahar, C.H. Chou (1995) Allometric scaling of grip strength by body mass in college-age men and women. Res. Q. Exerc. Sport, 66: 80-84.

23. Vanderburgh P.M., F.I. Katch (1996) Ratio scaling of $\mathrm{VO}_{2}$ max penalizes women with larger percent body fat, not lean body mass. Med. Sci. Sports Exerc., 28(9): 1204-1208.
Received 16.07.2013

Accepted 18.11.2013

(C) University of Physical Education, Warsaw, Poland

Acknowledgments

The study was financed by the Fund for the Development of Physical Culture. 